\title{
LETTER T O THE EDITOR
}

\section{SURVEILLANCE OF ANTI TUBERCULAR DRUG RESISTANCE}

Dear Editor,

In the April- June 2002 issue of JNMA, the article on "surveillance of anti tubercular drug resistance", G C G, Rijal B and Sharma AP had highlighted drug resistance in Nepal. ${ }^{1}$

According to the study, there were hundred percent multi drug resistances in previously treated cases. While mono resistance was 1.4 percent. Was there no mono resistance to $\mathrm{INH}$, rifampicin or streptomycin?

The authors conclude a relatively high mono resistance in new cases and a high level of multi drug resistance (MDR) in previously treated cases.

It is worthwhile to note that only four previously treated cases were included in the study. Such a small sample size could give an erroneously high figure regarding MDR tuberculosis in previously treated cases. In addition, a mono resistance of 1.4 percent seems to be a relatively low level of resistance.

In addition, the number of patients was too small to comment on the reasons for emerging multidrug resistance in case of previously treated cases.

It was unclear whether the German Nepal Tuberculosis Project's study had included patients previously treated for tuberculosis.
The WHO and IUALTD study was a report on prevalence of resistance to four first line drugs in 35 countries. ${ }^{2}$ While the study at TUTH had seventy new cases and only four previously treated cases. Hence, comparison would not give a meaningful result

About thirty percent of world's population has latent tuberculosis. Roughly, nine million cases of active tuberculosis emerge annually, resulting in 23 million deaths. There has been a worldwide increase in multiple drug resistant tuberculosis mainly because of immigration, HIV/AIDS, and the neglect of tuberculosis control programmes., ${ }^{3,4}$

While evaluating drug resistance, it is essential to have standard methodology for meaningful comparison among various institutes and countries. Focus should be emphasized on uniform laboratory methods, external and internal quality control, reliable drugs for setting up drug susceptibility, standard chemical in preparation of media in addition to a carefully elicited history and adequate representative sample

The level of initial drug resistance is considered as an epidemiological indicator to assess the degree of transmission of resistant tuberculosis in a community. In addition, it would serve as a useful tool for evaluating the national tuberculosis programmes. 5

There is an urgent need for conducting a welldesigned large-scale study to find the incidence and 
prevalence of primary as well as mono and multi drug resistant tuberculosis in the country.

New effective drugs with acceptable adverse effects are unlikely to be freely available in the near future in Nepal. Hence, the key to successful management of tuberculosis lies in adequate case finding and instigation of proper treatment that not only encompasses a standard regimen but also ensures compliance with and response to treatment for prevention of multi drug resistance

\section{Dr AA Latheef,}

MD, Resident in Internal Medicine,

BIR Hospital, Kathmandu.

(Dr. Latheef is from Maldives)

\section{REFERENCE}

1. GC G, R ijal B and S harma A P. S urveillance of anti tubercular drug resistance. J N M A A pril-J une 2002 41; 311-313.

2. T heW H O / U A T L D antituberculosis drug resistance in the world. W HO / U A L T D G lobal P roject on A nti- T uberculosis D rug R esistance $S$ urveillance 1994-1997. W H O document W H O /T B / 97.229.

3. H utchison D C, D robniewski F A, M il burn H J . M anagement of multipledrug-resistant tubercul osis. $R$ espir $M$ ed 2003 J an; 97(1): 65-70.

4. $R$ aviglione $M C, S$ nider $D E J, K$ ochi $A, G$ lobal epidemiology of tuberculosis: morbidity and mortality of a worldwide epidemic. J A M A 1995; 273: 220-226.

5. T he W H O /I U A L T D working group on antitubercul osis drug resistance surveillance. A ntitubercul osis drug resistance: is it worth measuring? M onaldi A rch C hest $D$ is 1996; 51:299-302. 